\title{
ADHESIVOS TIPO POLIURETANO OBTENIDOS A PARTIR DE ACEITE DE RICINO Y ALMIDÓN QUÍMICAMENTE MODIFICADOS
}

\author{
Manuel F. Valero \\ Programa de Ingeniería Química, Universidad de La Sabana, Campus Universitario del Puente del Común, Autopista Norte de \\ Bogotá, D.C., Apartado: 140013, Chía, Colombia
}

Recebido em 10/8/12; aceito em 5/12/12; publicado na web em 21/3/13

\begin{abstract}
POLYURETHANE ADHESIVES FROM CASTOR OIL AND MODIFIED STARCH. This study reports the preparation of polyurethane adhesives using polyols obtained from castor oil modified by a transesterification reaction with pentaerythritol and starch modified by glycosylation. The physical properties of the polyols such as hydroxyl value were determined and the infrared spectroscopic analysis of the polyols reported. The effect of varying the hydroxyl value in the polyols on physical properties of polyurethane coatings on wood and steel panels was determined. The characterization of polyurethane coatings carried out by IR spectroscopic analysis, scratch hardness resistance, impact resistance, lap shear strength, T-peel strength measurements, solvent resistance and chemical resistant determination were reported.
\end{abstract}

Keywords: adhesives; polyurethane; renewable resources.

\section{INTRODUCCIÓN}

Alrededor del $90 \%$ de los polioles utilizados actualmente para la producción de poliuretanos (PU) en todo el mundo están basados en poliéteres derivados a partir de etileno y óxido de propileno. Sin embargo, debido a la crisis del petróleo y el calentamiento global hay gran interés en la utilización de los recursos renovables como sustitutos de los derivados petroquímicos. Entre estos, los aceites vegetales han llamado la atención debido a sus ventajas como disponibilidad, sostenibilidad, versatilidad en la estructura y propiedades y relativo bajo costo. Adhesivos a base de urea-formaldehido y fenol-formaldehido se utilizan comúnmente para substratos como madera y acero, pero son muy sensibles a la hidrólisis y poco resistentes a la tensión. Estos adhesivos también producen riesgos para la salud debido a que liberan formaldehido. Adhesivos de poli acrilato también se utilizan para madera y acero, pero tienen algunas desventajas como un menor tiempo de vida útil, mayor costo y duración limitada. Por otro lado, existen polímeros que han sido tradicionalmente utilizados como adhesivos, tales como resinas epoxi, que presentan buenas propiedades físicas y buena resistencia al ataque por solventes, pero en su mayoría son de precio mucho más elevado o de difícil procesado. ${ }^{1,2}$

Para superar tales problemas, los científicos están tratando de desarrollar nuevos polímeros adhesivos. El poliuretano (PU) es una clase de polímero que se utiliza en recubrimientos, artículos de elastómeros, espumas y adhesivos. Los adhesivos de poliuretano son los más ampliamente utilizados debido a sus propiedades tales como versatilidad, flexibilidad, buen comportamiento a baja temperatura y alta resistencia. Las aplicaciones incluyen calzado, embalaje, componentes de automóviles y montaje de muebles. Las propiedades de los adhesivos de poliuretano pueden ser obtenidas a medida para adaptarse a una aplicación específica debido a la gran variedad de materias primas que pueden utilizarse en la formulación de poliuretanos. Aunque durante años los investigadores han estado tratando de desarrollar recubrimientos de poliuretano y redes interpenetradas de polímero (IPNs) a partir de aceites, la mayoría de estos se obtiene a partir de productos químicos sintéticos. Se han realizado pocos

*e-mail: manuel.valero@unisabana.edu.co estudios con relación a la preparación de poliuretanos a partir de materiales renovables y su aplicación como adhesivos.

El objetivo del trabajo fue desarrollar un adhesivo de poliuretano a partir de recursos naturales (aceite de ricino y almidón de yuca). Se buscó evaluar el cambio en la adhesión interfacial entre el substrato - en este caso madera y acero - y el poliuretano, según la estructura molecular y densidad de entrecruzamiento del poliuretano en función de la estructura y funcionalidad del poliol utilizado en la síntesis y relacionar la morfología de adhesión con la estructura química y densidad de entrecruzamiento del poliuretano a través del estudio de la propiedades mecánicas y de adhesión. Se pretende desarrollar adhesivos de poliuretano utilizando recursos renovables que son biodegradables y, la posibilidad de combinar su biodegradabilidad con la reducción de costos y las necesidades del mercado. ${ }^{3}$

\section{PARTE EXPERIMENTAL}

\section{Materiales}

Aceite de ricino (índice de hidroxilo: $163 \mathrm{mg} \mathrm{KOH} / \mathrm{g}$ e índice de acidez: 2,33 mg KOH/g) grado USP, distribuido por laboratorios León, Bucaramanga, Colombia. Pentaeritritol marca Fisher, distribuido por Produquímica de Colombia S.A. El almidón utilizado fue almidón de yuca, marca Maicena distribuido por Disa en Cali, Colombia. Los reactivos utilizados para la caracterización fueron de grado analítico marca Aldrich, distribuidos por Arquilab Ltda.

\section{MÉTODOS}

\section{Modificación del aceite de ricino por transesterificación con pentaeritritol}

La transesterificación del aceite de ricino usando pentaeritritol se realizó en un balón reactor de cuatro bocas equipado con termómetro, agitador mecánico, atmósfera inerte y condensador de reflujo. El balón reactor se cargó con el aceite de ricino, pentaeritritol y óxido de plomo ( $\mathrm{PbO}$ ), en relación $0.05 \%$ ( $\mathrm{PbO} /$ masa de aceite). La reacción se llevó a cabo a $210{ }^{\circ} \mathrm{C}$, por $2 \mathrm{~h}$. Los polioles penta $0(\mathrm{P} 0)$ que contiene $1.32 \%$ de pentaeritritol y penta 1 (P1) que contiene $2.64 \%$ de pentaeritritol (porcentaje en masa), respectivamente. Los 
polioles obtenidos se caracterizaron mediante la determinación del índice de hidroxilo (ASTM D1957-86) y espectroscopia infrarroja de transformada de Fourier (FTIR) en un espectrofotómetro Nicolet Avatar 360

\section{Modificación del almidón por glucosilación}

El almidón de yuca comercial reaccionó con el etilenglicol, en presencia de ácido sulfúrico como catalizador en una proporción $0.5 \%$ peso de catalizador/peso de etilenglicol (Figura 1S, material suplementario). Se utilizó un balón-reactor de cuatro bocas equipado con termómetro, agitador mecánico y entrada de nitrógeno. La reacción de glucosilación se llevó a cabo durante $2 \mathrm{~h}$, a $110 \pm 5{ }^{\circ} \mathrm{C}$ y $150 \pm 5$ mbar. Luego se adicionó óxido de bario. El óxido de bario reaccionó con el ácido sulfúrico y el producto de la reacción precipitó; este precipitado se separó por filtración. El contenido residual de etilenglicol después de la reacción se separó por destilación al vacío a $110 \pm 5{ }^{\circ} \mathrm{C}$ y $125 \pm 5$ mbar. El mismo procedimiento se utilizó para la reacción de glucosilación del almidón con glicerol.

\section{Síntesis de los poliol-glucósidos}

El glucósido de etilenglicol (GE) se mezcló con los polioles derivados del aceite de ricino en cantidades de 5 y $10 \%$ (peso de glucósido/peso de poliol) con una cantidad de catalizador (óxido de plomo) de $0.05 \%$ durante $2 \mathrm{~h}$ a $210 \pm 5{ }^{\circ} \mathrm{C}$, bajo atmósfera de nitrógeno, en un reactor similar usado para la transesterificación del aceite de ricino con pentaeritritol (Figura 2S, material suplementario). Se siguió el mismo procedimiento cuando se utilizó glucósido de glicerol (GG). Según el tipo de glucósido utilizado en la síntesis del poliol-glucósido, los productos obtenidos se denominaron poliol-glucósido de etilenglicol (PGE) y poliol-glucósido de glicerol (PGG) (Tabla 1).

Tabla 1. Índice de hidroxilo de los poliol-glucósidos de etilenglicol (GE) y glicerol (GG), respectivamente

\begin{tabular}{cc}
\hline \multicolumn{1}{c}{ Código de la muestra } & Índice de hidroxilo \\
\hline Aceite de ricino (CO) & $160 \pm 3$ \\
Poliol 0 (P0) (contiene 1.32\% en masa de & $191 \pm 3$ \\
pentaeritritol) & $236 \pm 3$ \\
Poliol 1 (P1) (contiene 2.64\% en masa de & \\
pentaeritritol) & $243 \pm 3$ \\
\hline Poliol-glucósido de etilenglicol (GE) & $266 \pm 3$ \\
\hline CO-GE-5\% & $287 \pm 3$ \\
P0-GE-5\% & $303 \pm 3$ \\
P1-GE-5\% & $342 \pm 3$ \\
CO-GE-10\% & $350 \pm 3$ \\
P0-GE-10\% & \\
P1-GE-10\% & $258 \pm 3$ \\
\hline Poliol-glucósido de glicerol (GG) & $284 \pm 3$ \\
CO-GG-5\% & $297 \pm 3$ \\
P0-GG-5\% & $338 \pm 3$ \\
P1-GG-5\% & $349 \pm 3$ \\
CO-GG-10\% & \\
P0-GG-10\% & \\
P1-GG-10\% & \\
\hline
\end{tabular}

\section{Sintesis de los adhesivos}

El poliol-glucósido se mezcló con diisocianato de isoforona (IPDI). El producto se agitó por 5 min para obtener una mezcla homogénea. El prepolímero obtenido se sometió a vacío y luego se aplicó sobre las superficies. La solución se aplicó con una brocha en el substrato con un espesor de $0.1 \mathrm{~mm}$ (ASTM D4147-93). Este proceso se realizó por triplicado. El período de curado constó de dos etapas, la primera a temperatura ambiente y la segunda a $90{ }^{\circ} \mathrm{C}$, ambas durante $4 \mathrm{~h}$. Se utilizó para la síntesis de los poliuretanos una relación $\mathrm{NCO} / \mathrm{OH}=1$.

\section{Caracterización de los adhesivos}

Los adhesivos de poliuretano se prepararon sobre madera disponible comercialmente (Clathrotropis brachypetala) y bandas de acero al carbono (acero de calibre 22) de $25 \times 300 \times 3 \mathrm{~mm}$. Las piezas fueron pulidas con papel de lija de grano de número 60 $(250 \mu \mathrm{m})$. Los adhesivos se aplicaron sobre paneles de ensayo y se dejaron secar a temperatura ambiente durante 7 días para asegurar un curado completo. La solución de adhesivo se aplicó con un pincel en ambos substratos, madera y acero, con un espesor entre 80-100 $\mu \mathrm{m}$ (el espesor de estos recubrimientos se midió por micrómetro digital). La estructura química del prepolímero se caracterizó utilizando espectroscopia infrarroja con transformada de Fourier en un espectrofotómetro Nicolet Avatar 360.

A los paneles revestidos se les caracterizó mediante la resistencia al rayado (dureza) según la norma BS 3900, resistencia al impacto que se determinó de acuerdo con la norma ASTM especificación D2794, ensayos de tracción y cizalladura que se realizaron en una máquina Zwick UTM modelo 1445 de acuerdo con la norma ASTM 906-82, ensayos de adherencia (ASTM 2651-01) y ensayos de resistencia a la corrosión y pruebas de resistencia al ataque por solventes (ASTM D543-87). Las pruebas de corrosión se realizaron en ácido $(\mathrm{HCl}$ $0,1 \mathrm{M})$, el álcali $(\mathrm{NaOH} 0,1 \mathrm{M})$ y $\mathrm{NaCl}(5 \%$ en peso de $\mathrm{NaCl})$. Los paneles recubiertos se sumergieron en los medios anteriormente mencionados a $80{ }^{\circ} \mathrm{C}$ durante $1 \mathrm{~h}$ y después se secó a temperatura ambiente durante $24 \mathrm{~h}$. El examen se llevó a cabo por la evidencia visual de ablandamiento, reducción, desprendimiento o decoloración de la película. ${ }^{4}$

Los análisis térmicos dinamo-mecánicos (DMTA) de los poliuretanos seleccionados se llevaron a cabo en un V. Rheometrics DMTA, modo cantiléver. Los ensayos se realizaron con un barrido de temperatura desde -150 a $170{ }^{\circ} \mathrm{C}$ a una frecuencia de $1 \mathrm{~Hz}$ y deformación de $0.1 \%$. La velocidad de calentamiento para todas las pruebas fue de $5{ }^{\circ} \mathrm{C} / \mathrm{min}$. Se utilizó una purga de nitrógeno de $60 \mathrm{~mL} / \mathrm{min}$. Todas las muestras analizadas tenían aproximadamente las mismas dimensiones de $2 \times 0,6 \times 0,3 \mathrm{~cm}$. El módulo de almacenamiento $\left(\mathrm{E}^{\prime}\right)$ y el factor de pérdida $(\tan \delta)$ se midieron. La temperatura correspondiente del máximo de la posición del pico tan $\delta$ fue tomada como la temperatura de transición vítrea $(\mathrm{Tg})$.

\section{RESULTADOS Y DISCUSIÓN}

\section{Caracterización de los poliol-glucósido}

En la Figura 1 se muestra el espectro infrarrojo del glucósido de glicerol comparado con el espectro de la mezcla física glicerol+almidón. Se observa un incremento en la banda correspondiente a los grupos $-\mathrm{OH}$ a una longitud de onda de $3400 \mathrm{~cm}^{-1}$. Adicionalmente, la mezcla física no muestra ningún pico significante entre una longitud de onda de 1100 y $1000 \mathrm{~cm}^{-1}$, mientras que el glucósido de glicerol si los registra. Un detalle de este espectro entre una longitud de onda de 1200 y $700 \mathrm{~cm}^{-1}$ se muestra en la Figura 3b. Estos picos están relacionados a la banda de absorción de los grupos O-C-O que se forman debido a la glucosilación del almidón. Por otro lado, los ensayos de iodometría realizados al glucósido de etilenglicol (GE) y al glucósido de glicerol (GG) no revelaron la presencia de cadenas de almidón (no se observó el color violeta característico en estos ensayos cuando se detecta la presencia de almidón). 

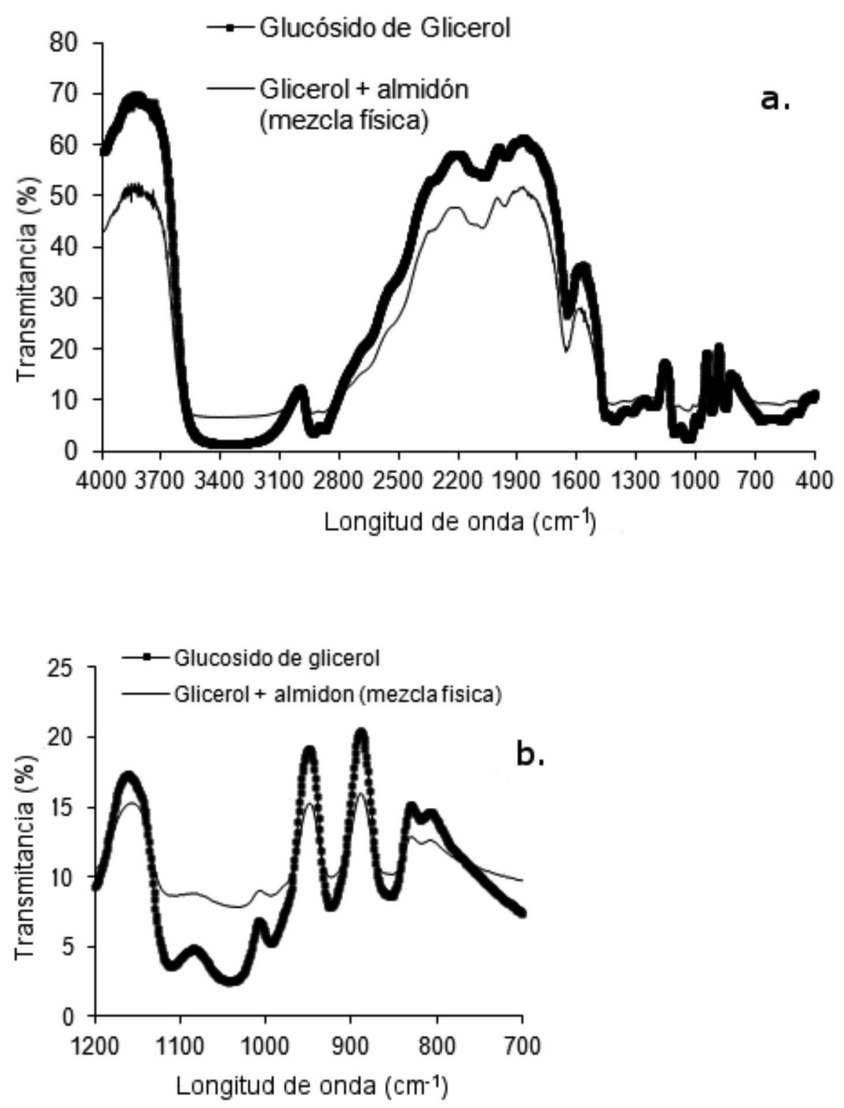

Figura 1. a. Espectro FTIR del glicerol glucósido y la mezcla física glicerol + almidón y b. detalle del espectro entre una longitud de onda de 1200 y $700 \mathrm{~cm}^{-1}$

En la Figura 2 se presenta el espectro FTIR del poliol-glucósido preparado a partir del poliol penta 1 (P1) con un contenido de glucósido de glicerol del 10\%, comparado con el espectro del poliol P1. El poliol-glucósido muestra un incremento en la banda correspondiente a los grupos $-\mathrm{OH}$ a una longitud de onda de 3400 $\mathrm{cm}^{-1}$ relacionado con el incremento en la funcionalidad del poliol. Se observa también un incremento en la banda correspondiente al grupo O-C-O a una longitud de onda de $1200 \mathrm{~cm}^{-1}$. El cambio en la banda asociada con el grupo O-C-O se debe a la presencia de los glucósidos resultado de la modificación química del almidón. Estos resultados evidencian la obtención de un nuevo poliol (poliolglucósido) y confirma el porqué se obtienen altos valores del índice

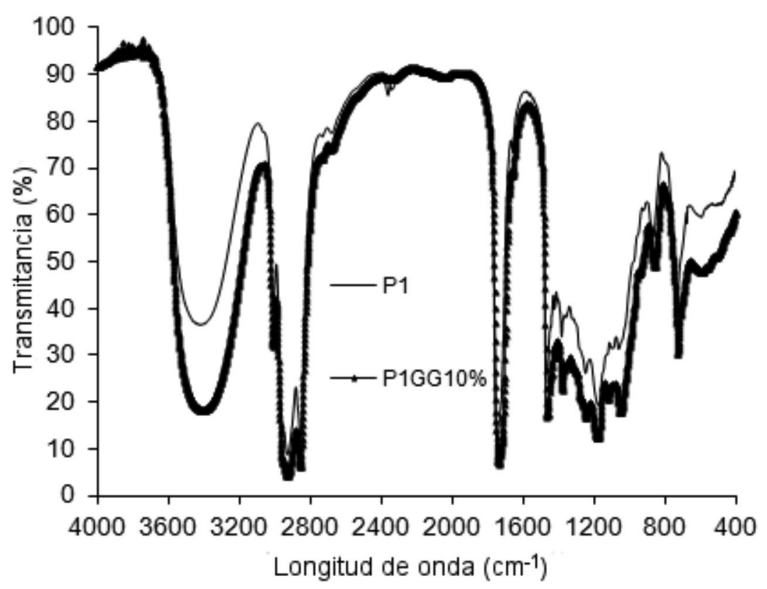

Figura 2. Espectro infrarrojo FTIR del poliol penta 1 (P1) y del poliol-glucósido (P1GE-10\%) preparado del poliol P1 y contiene 10\% del glucósido de glicerol de hidroxilo de los poliol-glucósido, debido a la modificación química del almidón por glucosilación.

En la Tabla 1 se muestra el índice de hidroxilo de los poliolglucósidos preparados a partir del aceite de higuerilla $(\mathrm{CO})$, polioles penta $0(\mathrm{P} 0)$ y penta $1(\mathrm{P} 1)$, respectivamente. Se encontró que el índice de hidroxilo es mayor cuando el poliol (CO, P0 o P1) reacciona por transesterificación con el glucósido de glicerina (GG) que con el glucósido de etilenglicol (GE), debido a que la funcionalidad del glucósido de glicerol es mayor que la del glucósido de etilenglicol. Se observa que al aumentar el contenido de glucósido del 5 al 10\% durante la transesterificación, el índice de hidroxilo - en todos los casos - aumenta. De igual forma, se muestra que el índice de hidroxilo es mayor para el poliol P1-glucósido, que para el correspondiente poliol P0-glucósido y este es mayor que el aceite-glucósido.

Caracterización de los adhesivos tipo poliuretano obtenidos a partir de los poliol-glucósidos

Se puede observar en las Tablas 2 y 3 que el PU-P1-GG mostró mejor resistencia al rayado que el PU-P0-GG y este que el PU-COGG. Este resultado obedece al alto grado de ramificación en la red PU-P1-CO que aumenta la densidad de entrecruzamiento lo que hace a la película más dura. En el caso del PU-CO-GG los grupos NCO están más distantes en comparación a la PU-P1-GG, por tanto la densidad de entrecruzamiento del PU-CO-GG es baja. Esto implica que la modificación del poliol por transesterificación con el glucósido aumenta la densidad de entrecruzamiento del adhesivo de poliuretano. Tendencia similar se observó también para la resistencia al impacto de los adhesivos de poliuretano anteriores. La resistencia al impacto aumenta de manera proporcional con el aumento del contenido de glucósido. La resistencia al impacto y la capacidad de flexión de los adhesivos dependen principalmente de la densidad de entrecruzamiento de los poliuretanos. Los valores de resistencia al impacto varían en el orden de PU-P1-GG-10\%> PU-P1-GG-5\%> PU-CO-GG-5\%. ${ }^{4}$

En las Tablas 2 y 3 se encuentran los resultados de los ensayos de tracción y cizalladura. Se observa que la el esfuerzo de tracción y cizalladura aumenta en los poliuretanos obtenidos a partir de los poliol-glucósidos en función: del incremento del porcentaje de glucósido; del tipo de glucósido: los poliol-glucósido de glicerol (P1-GG) exhiben mayor resistencia a la tracción y cizalladura que los poliuretanos obtenidos a partir de los poliol-glucósido de etilenglicol (P1-EG); del tipo de poliol: los poliol 1-glucósido de glicerol (P1GG) exhiben mayor resistencia a la tracción y cizalladura que los poliuretanos obtenidos a partir de los poliol 0-glucósido de glicerol (P0-GG) y poliuretanos obtenidos a partir de los aceite-glucósido de glicerol (CO-GG).

Las resistencias al adhesión los materiales se determinaron y los resultados se muestran en las Tablas 2 y 3 . Como se esperaba los adhesivos obtenidos a partir del poliol-glucósido P1-GG presentan mayores valores de esfuerzo de adhesión. Se observa que la resistencia a la adhesión de los materiales aumenta con el aumento en el contenido de glucósido. A medida que aumenta el contenido de glucósido (es decir, una mayor densidad de entrecruzamiento), aumentan la rigidez y la resistencia de la unión adhesiva. La adhesión entre el recubrimiento y el substrato se considera satisfactoria debido a que la falla se produce debido a un fallo cohesivo del substrato. Los adhesivos de poliuretano altamente entrecruzados tienen un mejor rendimiento de adhesión que los adhesivos de poliuretano con estructura menos reticulada. La alta funcionalidad hidroxilo del poliol proporciona puntos de reticulación necesaria para la formación de la red de polímero lo que genera una buena resistencia cohesiva. En su conjunto, mediante la comparación de los resultados obtenidos en las pruebas, el poliol P1-GG produjo los mejores adhesivos. Los 
Tabla 2. Propiedades mecánicas y de adhesión de los recubrimientos obtenidos a partir de los poliol-glucósido de etilenglicol $(\mathrm{relación} \mathrm{NCO} / \mathrm{OH}=1)$

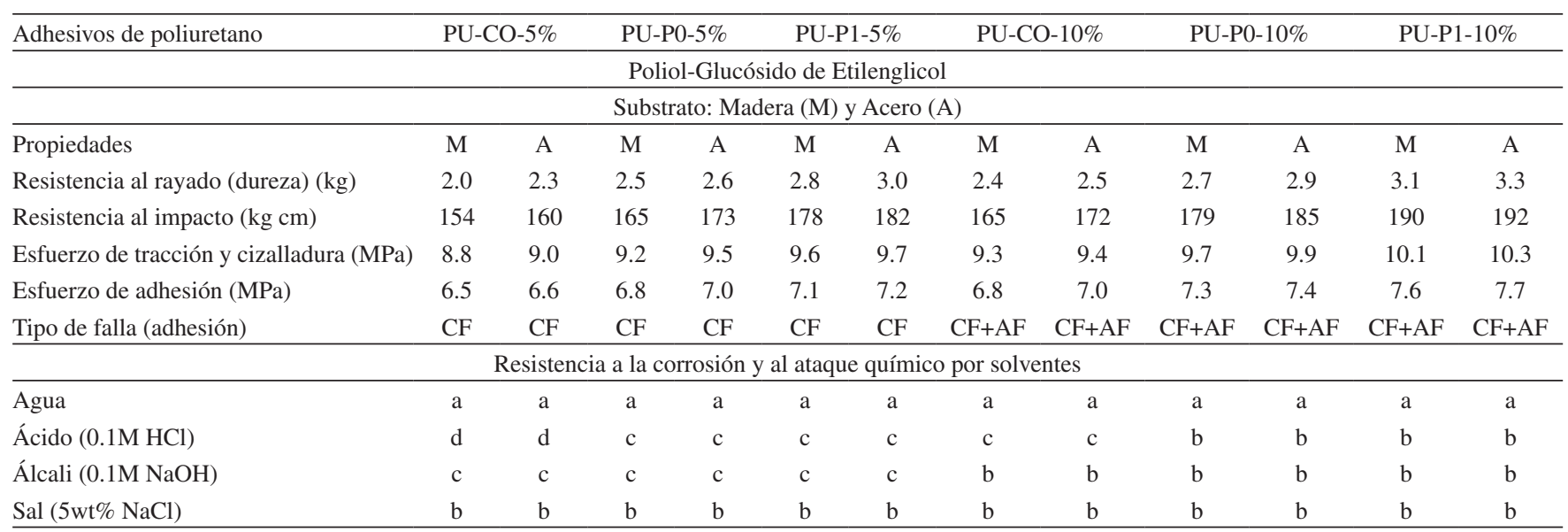

CF: falla cohesiva del adhesivo; AF: falla de adhesión; SF: falla del substrato. a, No afectado; b, pérdida de brillo; c, pérdida de brillo y peso; d, remoción parcial de la película de adhesivo.

Tabla 3. Propiedades mecánicas y de adhesión de los recubrimientos obtenidos a partir de los poliol-glucósido de glicerol (relación NCO/OH = 1)

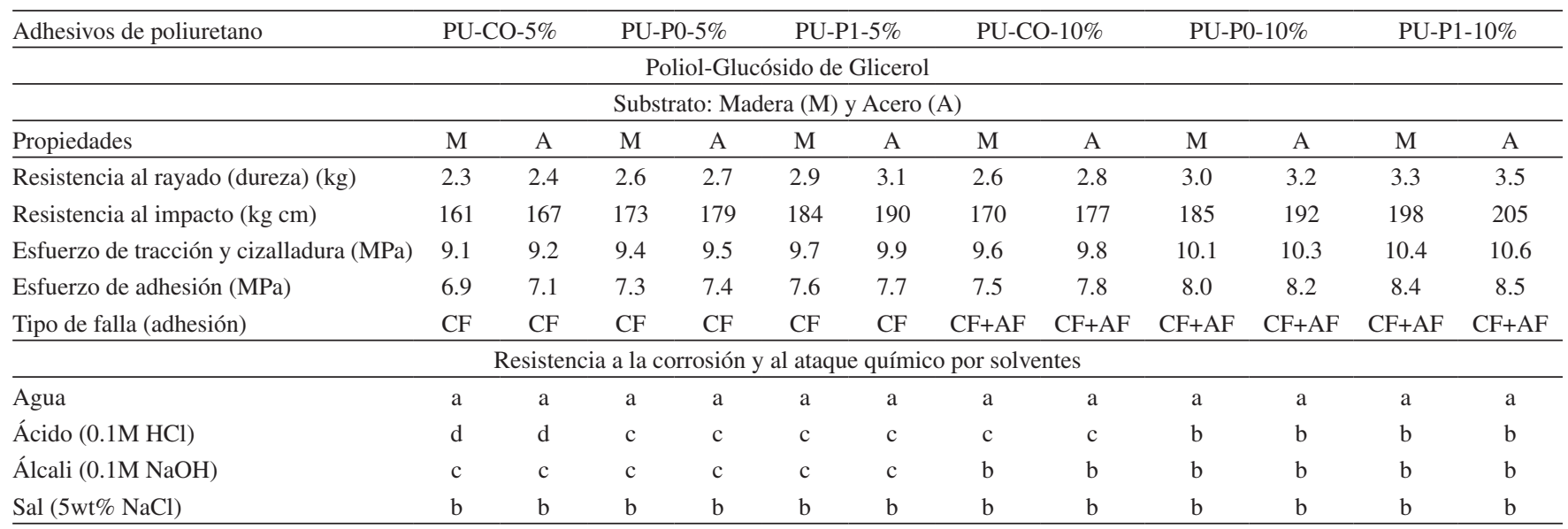

CF: falla cohesiva del adhesivo; AF: falla de adhesión; SF: falla del substrato. a, No afectado; b, pérdida de brillo; c, pérdida de brillo y peso; d, remoción parcial de la película de adhesivo.

resultados mostraron que la adherencia entre el adhesivo/madera fue menor que la cohesión del adhesivo/acero.,

Todos los adhesivos de poliuretano fueron sometidos a exposición en medios químicos diferentes para estudiar su comportamiento de resistencia a la corrosión; los cambios en su apariencia, peso, la adhesión han sido tabulados en las Tablas 2 y 3 . Se observó además que los paneles recubiertos presentan pérdida de brillo en cuando se expone al ácido ( $\mathrm{HCl} 0,1 \mathrm{M})$, el álcali $(\mathrm{NaOH} 0,1 \mathrm{M})$ y $\mathrm{NaCl}(5 \%$ en peso de $\mathrm{NaCl}$ ). De los resultados proporcionados se puede deducir que al aumentar el contenido de glucósido no se tuvo ningún efecto significativo en el comportamiento de la resistencia al ataque químico de los adhesivos. ${ }^{7}$

Los termogramas de los poliuretanos obtenidos a partir de los poliol-glucósido CO-GG-5\% y P1-GG-5\% se muestran en la Figura 3. Se observan tres regiones: la primera que corresponde a la degradación de componentes minoritarios del aceite de ricino y a la descomposición de las unidades D-glucopiranosil del almidón modificado. La segunda que corresponde a la degradación de los enlaces de uretano de los segmentos duros y la tercera región que corresponde a la degradación de los segmentos suaves del poliuretano. Las temperaturas de descomposición para varios porcentajes de pérdida de peso se muestran en la Tabla 4. Se observa que la estabilidad térmica varía de la siguiente manera: PU-P1-GG-10\%>
PU-P0-GG-10\% > PU-CO-GG-10\%. Se observa también que los poliuretanos que contienen glucósido de glicerol poseen mayor estabilidad térmica que los poliuretanos que contienen glucósido de etilenglicol (P0-GG-10\% > P0-EG-10\%). Este resultado confirma que al aumentar la densidad de entrecruzamiento del poliuretano aumenta la estabilidad térmica. ${ }^{8}$

El módulo $E^{\prime}$ de los poliuretanos obtenidos a partir de los poliol-glucósido del aceite de ricino, penta 0 y penta 1 , varia de la siguiente manera, de mayor a menor: PU de P1-GG-10\% >PU de P0-GG-10\% > PU de CO-GG-10\%, Figura 4a. Al aumentar la funcionalidad del poliol aumenta a densidad de entrecruzamiento del PU, por lo tanto el módulo de almacenamiento aumenta como respuesta al esfuerzo aplicado (es decir el material es más rígido a una frecuencia y temperatura constantes). En la Figura $4 \mathrm{~b}$ se observa que la temperatura de transición vítrea $(\mathrm{Tg})$ es mayor para el PU obtenido del P1GG10\%; se observa también que la intensidad del pico de tan(delta) para este poliuretano disminuye (la restricción en la movilidad de las cadenas aumenta debido al incremento en el grado de entrecruzamiento del PU). Estos resultados evidencian la tendencia al aumento de la rigidez de los materiales obtenidos a partir de los poliol-glucósidos, debido al incremento en la densidad de entrecruzamiento del poliuretano en función del índice de hidroxilo del poliol utilizado en la síntesis. ${ }^{9}$ 

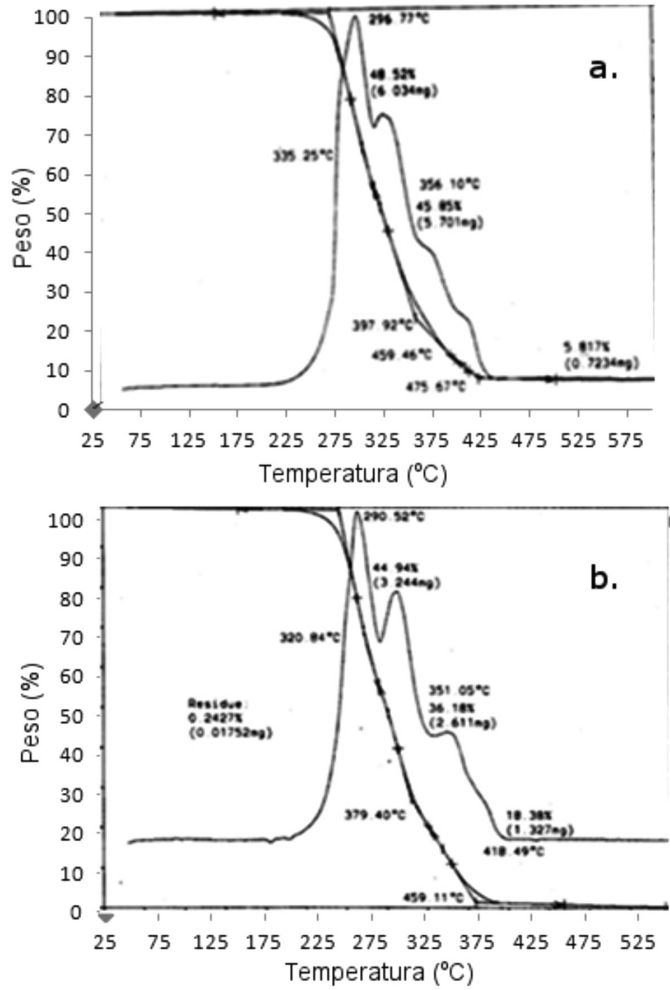

Figura 3. Termogramas TGA de los poliuretanos (relación $\mathrm{NCO} / \mathrm{OH}=1$ ) a. $P U-C O-G G-5 \%$ (aceite de ricino con $5 \%$ de glucósido de glicerol) y b. PUP1-GG-5\% (penta 1 con $5 \%$ de glucósido de glicerol)

Tabla 4. Temperaturas de descomposición de algunos de los adhesivos de poliuretanos obtenidos a partir de los poliol-glucósido

\begin{tabular}{|c|c|c|c|c|}
\hline \multirow{2}{*}{$\begin{array}{l}\text { Adhesivos de } \\
\text { poliuretano } \\
\text { PU-CO-GE-10\% }\end{array}$} & \multirow[b]{2}{*}{$\mathrm{Ti}$} & \multirow{2}{*}{$\begin{array}{c}\begin{array}{c}\text { Temperatura } \\
\left({ }^{\circ} \mathrm{C}\right)\end{array} \\
288.03\end{array}$} & \multicolumn{2}{|c|}{ \% pérdida de peso } \\
\hline & & & & \\
\hline & $\operatorname{Tr} 1$ & 334.08 & \multicolumn{2}{|c|}{53.86} \\
\hline & $\operatorname{Tr} 2$ & 356.5 & \multicolumn{2}{|c|}{42.36} \\
\hline & $\operatorname{Tr} 3$ & 464.36 & \multicolumn{2}{|c|}{3.886} \\
\hline & $\mathrm{Tf}$ & 476.72 & $\%$ Residuo & 0.2127 \\
\hline \multirow[t]{5}{*}{ PU-CO-GG-10\% } & $\mathrm{Ti}$ & 296.77 & & \\
\hline & $\operatorname{Tr} 1$ & 335.25 & \multicolumn{2}{|c|}{48.52} \\
\hline & $\operatorname{Tr} 2$ & 356.1 & \multicolumn{2}{|c|}{45.85} \\
\hline & $\operatorname{Tr} 3$ & 459.46 & \multicolumn{2}{|c|}{5.817} \\
\hline & $\mathrm{Tf}$ & 475.67 & $\%$ Residuo & 0 \\
\hline \multirow[t]{5}{*}{ PU-P0-GG-10\% } & $\mathrm{Ti}$ & 289.64 & & \\
\hline & $\operatorname{Tr} 1$ & 322.59 & \multicolumn{2}{|c|}{43.72} \\
\hline & $\operatorname{Tr} 2$ & 351.15 & \multicolumn{2}{|c|}{36.64} \\
\hline & $\operatorname{Tr} 3$ & 444.88 & \multicolumn{2}{|c|}{19.69} \\
\hline & $\mathrm{Tf}$ & 462.37 & $\%$ Residuo & 0.0299 \\
\hline \multirow[t]{5}{*}{ PU-P0-GG-10\% } & $\mathrm{Ti}$ & 294.16 & & \\
\hline & $\operatorname{Tr} 1$ & 325.77 & \multicolumn{2}{|c|}{47.17} \\
\hline & $\operatorname{Tr} 2$ & 355.06 & \multicolumn{2}{|c|}{34.36} \\
\hline & $\operatorname{Tr} 3$ & 424.64 & \multicolumn{2}{|c|}{18.51} \\
\hline & $\mathrm{Tf}$ & 458.19 & $\%$ Residuo & 0.3181 \\
\hline \multirow[t]{5}{*}{ PU-P1-GE-10\% } & $\mathrm{Ti}$ & 290.47 & & \\
\hline & $\operatorname{Tr} 1$ & 334.63 & \multicolumn{2}{|c|}{60.74} \\
\hline & $\operatorname{Tr} 2$ & 360 & \multicolumn{2}{|c|}{24.67} \\
\hline & $\operatorname{Tr} 3$ & 430.51 & \multicolumn{2}{|c|}{15.03} \\
\hline & $\mathrm{Tf}$ & 465.4 & $\%$ Residuo & 0 \\
\hline
\end{tabular}

Ti: temperatura inicial de degradación; Tri: temperatura de degradación de las regiones 1,2 y 3 , respectivamente; Tf: temperatura final de degradación.
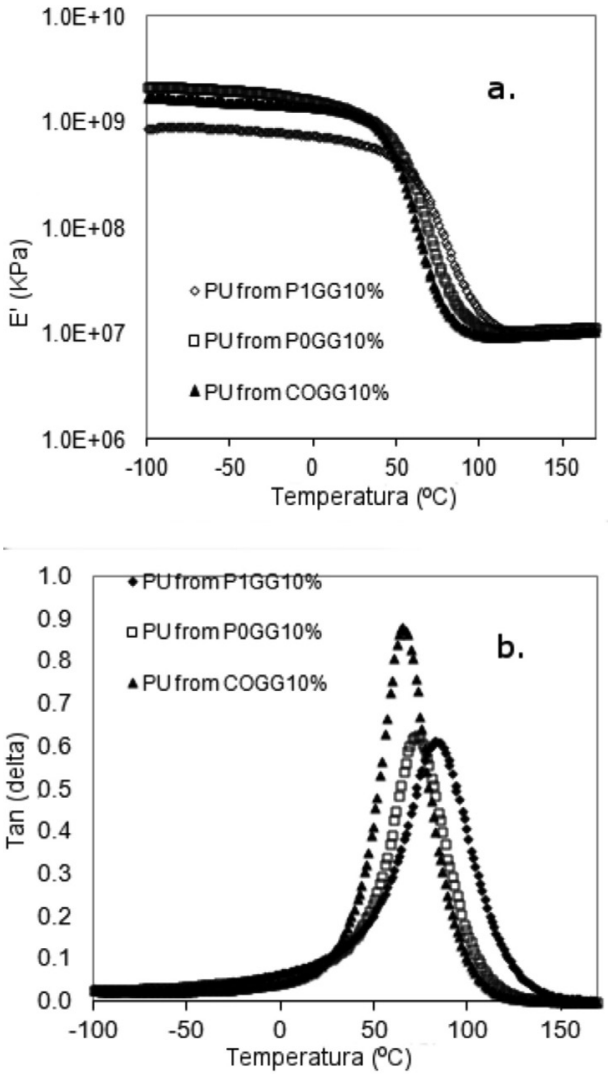

Figura 4. Curvas dinamo-mecánicas del poliuretano obtenido a partir del aceite de ricino-glucósido (COGG10\%), penta 0-glucósido (P0GG10\%), y el penta 1-glucósido (P1GG10\%) en función de la temperatura a. módulo de almacenamiento $E^{\prime} y$ b. $\tan ($ delta)

\section{CONCLUSIONES}

Adhesivos tipo poliuretano a partir de aceite de ricino modificado por una reacción de transesterificación con pentaeritritol y posterior reacción con el almidón de yuca modificado por glucosilación presentan mejores propiedades de adhesión en comparación con adhesivos de poliuretano de aceite de ricino original. Al aumentar el contenido de glucósido en los adhesivos se incrementaron las propiedades mecánicas y de adhesión. A medida que el índice de hidroxilo aumentó, las propiedades de los adhesivos aumentaron debido a la formación de una estructura más entrecruzada. Los adhesivos de poliuretano a partir de aceite de ricino modificado podrían ser utilizados como un producto eficaz protector para la corrosión - obtenido a partir de recursos renovables - candidato para el uso en un proyecto práctico, especialmente para la adhesión de los metales.

\section{MATERIAL SUPLEMENTARIO}

El material suplementario se puede consultar de manera libre en la página web http://quimicanova.sbq.org.br en forma de archivo PDF.

\section{AGRADECIMIENTOS}

A la Investigación Dirección de la Universidad de La Sabana por la asistencia financiera.

\section{REFERENCIAS}

1. Somani, K. P.; Kansara, S. S.; Patel, N. K.; Rakshit, A. K.; Int. J. Adhes. Adhes. 2003, 23, 269. 
2. Sandip, D.; Jigar, V. P.; Vijay, K. S.; Int. J. Adhes. Adhes. 2003, 23, 393.

3. Elena, O.-C.; Int. J. Adhes. Adhes. 2009, 29, 309.

4. Keyur, P. S.; Sujata, S. K.; Natvar, K. P.; Animesh, K. R.; Int. J. Adhes. Adhes. 2003, 23, 269.

5. Esposito, C.; Prinari, P.; Cannoletta, D.; Mensitieri, G.; Maffezzoli. A.; Int. J. Adhes. Adhes. 2008, 28, 91.
6. Akram, D.; Sharmin, E.; Ahmad, S.; Progr. Org. Coat. 2008, 63, 25.

7. Deepak, M.; Vijay, K. S.; Int. J. Adhes. Adhes. 2010, 30, 47.

8. Vinay, S.; Kundu, P. P.; Progr. Polym. Sci. 2008, 33, 1199.

9. Sandip, D.; Anurag, E.; Vijay, K. S.; J. Polym. Res. 2003, 10, 275. 